\title{
Article
}

\section{Alkaloids as Alternative Probes to Characterize the Relative Hydrophobicity of Aqueous Biphasic Systems}

Jorge Fernando Brandão Pereira, Agnes Magri, Maria V Quental, Maria Gonzalez-Miquel, Mara G. Freire, and Joao A.P. Coutinho

ACS Sustainable Chem. Eng., Just Accepted Manuscript • DOI: 10.1021/

acssuschemeng.5b01466 • Publication Date (Web): 26 Jan 2016

Downloaded from http://pubs.acs.org on February 1, 2016

\section{Just Accepted}

"Just Accepted" manuscripts have been peer-reviewed and accepted for publication. They are posted online prior to technical editing, formatting for publication and author proofing. The American Chemical Society provides "Just Accepted" as a free service to the research community to expedite the dissemination of scientific material as soon as possible after acceptance. "Just Accepted" manuscripts appear in full in PDF format accompanied by an HTML abstract. "Just Accepted" manuscripts have been fully peer reviewed, but should not be considered the official version of record. They are accessible to all readers and citable by the Digital Object Identifier (DOI®). "Just Accepted" is an optional service offered to authors. Therefore, the "Just Accepted" Web site may not include all articles that will be published in the journal. After a manuscript is technically edited and formatted, it will be removed from the "Just Accepted" Web site and published as an ASAP article. Note that technical editing may introduce minor changes to the manuscript text and/or graphics which could affect content, and all legal disclaimers and ethical guidelines that apply to the journal pertain. ACS cannot be held responsible for errors or consequences arising from the use of information contained in these "Just Accepted" manuscripts. 


\title{
Alkaloids as Alternative Probes to Characterize the Relative
} Hydrophobicity of Aqueous Biphasic Systems

\author{
Jorge F. B. Pereira ${ }^{1 *}$, Agnes Magri ${ }^{1}$, Maria V. Quental, ${ }^{2}$ Maria Gonzalez-Miquel ${ }^{3}$, Mara \\ G. Freire ${ }^{2}$, João A. P. Coutinho ${ }^{2}$ \\ ${ }^{1}$ Department of Bioprocess and Biotechnology, School of Pharmaceutical Sciences, \\ UNESP - Univ Estadual Paulista, Rodovia Araraquara-Jaú/Km 01 14801-902 - \\ Araraquara, SP, Brazil \\ ${ }^{2}$ CICECO - Aveiro Institute of Materials, Department of Chemistry, University of \\ Aveiro, 3810-193 Aveiro, Portugal \\ ${ }^{3}$ School of Chemical Engineering and Analytical Science, The University of \\ Manchester, Manchester, M13 9PL, United Kingdom
}

${ }^{*}$ Corresponding author

School of Pharmaceutical Sciences, UNESP - Univ Estadual Paulista, Rodovia Araraquara-Jaú/Km 01 14801-902 - Araraquara, SP, Brazil.

Tel: +55 (16) 3301-4675; e-mail address: jfbpereira@,fcfar.unesp.br 


\begin{abstract}
In order to overcome the lack of characterization on the relative hydrophobicity of aqueous biphasic systems (ABS), the partition of three alkaloids as alternative probes, was evaluated in a series of biocompatible ABS composed of cholinium-based salts or ionic liquids (ILs) and polyethylene glycol (PEG). The caffeine partitioning in ABS was firstly addressed to infer on the effect of the phase-forming components composition. In all systems, caffeine preferentially concentrates in the lower water content PEG-rich phase. Additionally, a linear dependence between the logarithmic function of the partition coefficients and the water content ratio was found. To confirm this linear dependency, the partition coefficients of caffeine, theobromine and theophylline were determined in other ABS formed by different cholinium-based salts/ILs. In most systems, it is shown that all alkaloids partition to the most hydrophobic phase. To support the experimental results, COSMO-RS (Conductor-like Screening Model for Real Solvents) was used to compute the screening charge distributions of both phaseforming components of ABS and alkaloids, the excess enthalpy of mixing and the activity coefficients at infinite dilution. It is here demonstrated that the partition trend of alkaloids can be used to address the relative hydrophobicity of the coexisting phases in polymer-salt/-IL ABS.
\end{abstract}

Keywords: hydrophobicity; aqueous biphasic systems; cholinium; ionic liquids; polyethylene glycol; alkaloid; caffeine; theobromine; theophylline. 


\section{Introduction}

Research on more biocompatible and sustainable purification methods led to the resurgence of interest in liquid-liquid extraction processes using Aqueous Biphasic Systems (ABS). This particular type of water-rich systems has been suggested as an effective alternative to conventional extraction methods using volatile organic solvents. ${ }^{1,2}$ ABS are water-based systems formed by combinations of compounds ranging from the most common polymer/polymer ${ }^{1,2}$ and polymer/salt ${ }^{1,2}$ to the recent ionic liquid (IL)/salt ${ }^{3,4}$, polymer/ $\mathrm{IL}^{4,5}$ and $\mathrm{IL} /$ carbohydrate $^{4,6}$ pairs of phase-forming components. Due to their low interfacial tension and high water content, ABS preserve the biological activity of biomolecules by the formation of a biocompatible aqueous environment similar to intracellular environments. ${ }^{7,8}$ Moreover, several classes of ABS are simpler and cheaper than most of chromatographic techniques used for separation approaches and are highly selective for a wide variety of solutes, enhancing thus their importance in biotechnological, chemical and pharmaceutical industries. ${ }^{2-4}$

Recently, the use of ILs in the formation of $\mathrm{ABS}^{3,4}$ increased the number of possible systems and applications for this type of extractions, mainly due to their excellent selectivity for extracting similar or complex solutes. ${ }^{4}$ ILs are ionic compounds with low melting points, exhibiting a series of interesting properties, some closely related with their application as phase-forming agents of ABS, in particular their large solvation capability and great tunability. ${ }^{4,9}$ Besides these important solvation characteristics of ILs, some environmental concerns about have been expressed, in particular for IL families based on imidazolium or pyridinium cations, and ILs with fluorinated anions, which present an undesirable level of toxicity and low biodegradability. ${ }^{10}$

According to the Green Chemistry principles, to be considered as an environmentally friendly compound an IL should accomplish a set of particular features, for instance, it should have minor accumulation and persistence in the environment (biodegradability), it should be based in renewable raw materials, be obtained by less hazardous and more economic synthesis, among others. ${ }^{11}$ Following these principles, recently, the appearance of several cholinium-based salts ([Ch $]^{+}$-salts), especially those with low melting points, has been attracting the attention of many researchers. ${ }^{12}$ In general, cholinium-based compounds are cheaper than traditional ILs, may be completely derived from renewable sources, and may present outstanding 
biodegradability and low toxicity. ${ }^{13}$ In this context, the use of cholinium-based compounds (salts or ILs) has been proposed as an alternative to increase the sustainable and biocompatible features of IL-based ABS. ${ }^{14,15,16}$ Herein, considering the importance of the fulfillment of several green chemistry principles, we propose the use of ABS composed of polyethylene glycol (PEG) polymers (inert and biocompatible) in combination with $[\mathrm{Ch}]^{+}$-salts or ILs.

Despite the large set of IL-based ABS described in the last decade ${ }^{4}$, these extractive platforms are not yet well understood, and the prediction of their tunability and selectivity is still a difficult task. Particularly, the behavior of ABS composed of ILs and water soluble polymers is quite complex, but far more interesting in terms of polarities and separation ranges achieved. ${ }^{5}$ In order to understand the mechanisms driving the phase-split of these systems, we have been carrying out some experimental and simulation studies for different sets of ABS. ${ }^{5,14,17,18}$ These works have shown that a combination of important interactions between all species in equilibrium (i.e., ILs ions, polymers and water), as hydrogen-bonding type interactions, and some hydration mechanisms (such as the "washing-out" mechanism) have a primordial role through phase separation. These works have been disclosing some of the complexity behind the PEG/ILs ABS formation, but their use for the separation of biomolecules is still residual, while the molecular interactions driving the partition of compounds on these systems is not yet entirely understood. ${ }^{5,19}$

In spite of the lack of understanding on the mechanisms underlying the selective separation of solutes in polymer/IL ABS, these systems appear as promising liquidliquid separation systems to be used at industrial scale, in particular coupled to centrifugal partition chromatography (CPC). CPC is a liquid-liquid chromatographic technique which allows the continuous selective separation of solutes. ${ }^{20}$ Nevertheless, the understanding of the solutes partition in PEG/ILs ABS is a preliminary requirement for the design of adequate systems envisaging their further use in CPC platforms. Thus, the main objective of this work consists on the evaluation of the partitioning of a series of alkaloids, here used to as relative hydrophobicity probes, to gauge the solutes partition mechanisms in a series of ABS composed of PEG (with an average molecular weight of 600 g.mol ${ }^{-1}$ ) and different cholinium-based compounds, ranging from salts, such as cholinium chloride ([Ch]Cl), to ILs, such as cholinium acetate ([Ch] [OAc]).

\section{Experimental Section}




\section{Materials}

Cholinium Chloride, $[\mathrm{Ch}] \mathrm{Cl}$, Cholinium Bicarbonate, $[\mathrm{Ch}][\mathrm{Bic}]$, Cholinium Dihydrogencitrate, [Ch][DHcit], and Cholinium Dihydrogenphosphate, [Ch][DHph] were acquired from Sigma-Aldrich ${ }^{\circledR}$ while Cholinium Acetate, [Ch][OAc], was purchased from Iolitec ${ }^{\circledR}$. All salts were dried under constant agitation and vacuum, at a moderate temperature $\left(80^{\circ} \mathrm{C}\right)$, for a minimum of $48 \mathrm{~h}$, to reduce the volatile impurities and water content to negligible values. After this procedure, ${ }^{1} \mathrm{H}$ and ${ }^{13} \mathrm{C}$ NMR spectra were performed to evaluate the purity of all salts. All salts showed mass fraction purities higher than $99 \%$.

PEG of an average molecular weight 600 g. $\mathrm{mol}^{-1}$ (abbreviated as PEG-600) was supplied by Fluka ${ }^{\circledR}$, and was used as received.

The water used was double distilled, passed across a reverse osmosis system and further treated with a Milli-Q plus 185 water purification apparatus.

The alkaloids theobromine (purity $\geq 99 \mathrm{wt} \%$ ) and theophylline (purity $\geq 99 \mathrm{wt} \%$ ) were supplied by Sigma-Aldrich. Caffeine (purity $\geq 99.5 \mathrm{wt} \%$ ) was purchased from José Manuel Vaz Pereira. All alkaloids were used without further purification, and their chemical structures are shown in Table 1.

\section{Methods}

\section{Partitioning of alkaloids}

All liquid-liquid partitioning systems were gravimetrically prepared in graduated centrifuge tubes (within an uncertainty of $\pm 10^{-4} \mathrm{~g}$ ). The selected mixtures (Table S1 and $\mathrm{S} 2$ in the Supporting Information) were prepared according to the corresponding ternary phase diagrams previously published by us. ${ }^{14}$ Aqueous solutions of caffeine at 0.0772 mol. $\mathrm{L}^{-1}$, theobromine at $0.0004 \mathrm{~mol} . \mathrm{L}^{-1}$, and theophylline at $0.0055 \mathrm{~mol} . \mathrm{L}^{-1}$ were used. All the solutions were prepared in order to guarantee that all alkaloids are at conditions close to infinite dilution aiming at avoiding solute-solute interactions and consequent influences on the alkaloids partitioning coefficients. After a complete dissolution of all components in the mixture by stirring, the mixture was left to equilibrate for $12 \mathrm{~h}$ immersed in a refrigerated water bath, at $25^{\circ} \mathrm{C}\left( \pm 0.01^{\circ} \mathrm{C}\right)$, to achieve the equilibrium on the partitioning of each alkaloid between the phases. After equilibration the phases were carefully separated and the alkaloids quantified in both top and bottom phases by UV spectroscopy using a SHIMADZU UV-1700, Pharma-Spec spectrometer, at $273 \mathrm{~nm}$. Calibration curves for the determination of each alkaloid were previously established at 
the respective maximum absorbance peaks. All the assays were performed in triplicate. In order to evaluate how the compound concentration influence the partition coefficient, a similar caffeine partitioning measurements were performed for three independent [Ch]Cl-based systems, in which different caffeine aqueous solutions at $0.0772 \mathrm{~mol} . \mathrm{L}^{-1}$, 0.0515 mol. $\mathrm{L}^{-1}$ and $0.0257 \mathrm{~mol} . \mathrm{L}^{-1}$ were used.

The alkaloids partition coefficients $(\mathrm{K})$ were estimated according to the following equation:

$\mathrm{K}=\frac{[\mathrm{alk}]_{\mathrm{PEG}}}{[\mathrm{alk}]_{\text {Salt }}}$

where $[\mathrm{alk}]_{\mathrm{PEG}}$ and $[\mathrm{alk}]_{\text {Salt }}$ represent the concentration of each alkaloid in the PEG600 and $[\mathrm{Ch}]^{+}$-salt-rich phases, respectively. It should be noted that the relative densities of salt and polymeric-rich phases are not constant, and the denser phase can be either PEG- or salt-rich phase, depending on the salt used ( $c f$. Table S2 in the Supporting Information).

\section{Water content measurements}

The water content of the top and bottom phases were measured by volumetric titration at $25^{\circ} \mathrm{C}$ using a Karl-Fischer 852 Titrando from Metrohm ${ }^{\circledR}$ (Switzerland), using HYDRANAL ${ }^{\circledR}$-Methanol Rapid (reagent for accelerated volumetric one-component KF titration) and HYDRANAL ${ }^{\circledR}$-Composite 5 (Reagent for volumetric one-component Karl Fischer titration - methanol free), both supplied by Sigma-Aldrich ${ }^{\circledR}$ as titrants.

\section{pH and conductivity measurements}

After the phase separation, the $\mathrm{pH}$ and conductivity of each phase was determined using a $\mathrm{pH}$ Meter (Seven Multi METTLER TOLEDO) at $25^{\circ} \mathrm{C}$, within an uncertainty of \pm 0.01 . 


\section{Results and Discussion}

\section{Partitioning of caffeine}

In order to understand the mechanisms behind the solutes partitioning in the PEG-600/[Ch $]^{+}$-salts ABS, and to explore their potential as novel extractive systems, the partitioning of caffeine was firstly addressed as a hydrophobicity probe. Caffeine is a xanthine alkaloid, that is an interesting molecule to be used as solute model, since it does not suffer speciation in a large range of $\mathrm{pH}$ values $\left(\mathrm{pK}_{\mathrm{a} 1} / \mathrm{pK}_{\mathrm{a} 2}=0.12 / 10.5\right)$ and it is only slightly hydrophilic (the logarithm of octanol/water partition coefficient, $\log ($ Kow $)=-0.07)^{21}$

Recently, we have shown that PEG-600 can form two immiscible phases with high melting $[\mathrm{Ch}]^{+}$-salts, as well with the low melting $[\mathrm{Ch}]^{+}$-salts, which can be defined as ILs. ${ }^{14}$ Thus, in this work, we selected two series of PEG-600-based ABS to assess the $[\mathrm{Ch}]^{+}$-salts' influence on the partitioning of caffeine, in particular, the effect of cholinium chloride $([\mathrm{Ch}] \mathrm{Cl})$ and cholinium acetate $([\mathrm{Ch}][\mathrm{OAc}])$ - corresponding to high melting and low melting salts, respectively. The first set of assays aimed at the evaluation of different compositions of polymer and salt/IL on the partitioning of caffeine at $25^{\circ} \mathrm{C}$. For each type of ABS, namely PEG-600 $+[\mathrm{Ch}] \mathrm{Cl}+\mathrm{H}_{2} \mathrm{O}$ and PEG-600 $+[\mathrm{Ch}][\mathrm{OAc}]+\mathrm{H}_{2} \mathrm{O}$, seven mixture points were prepared, as depicted in Figure 1. The detailed weight fraction compositions are provided in the Supporting Information (Table S1).

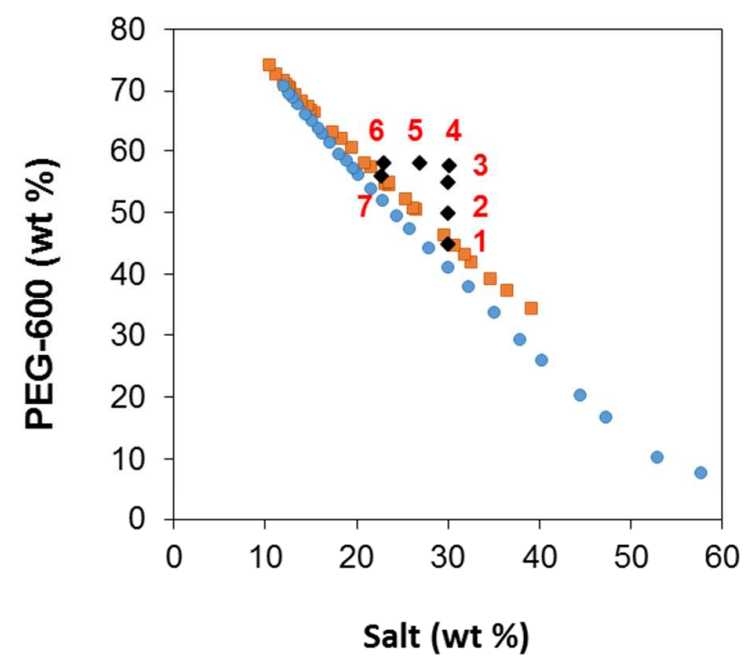

Figure 1. Experimental solubility data ${ }^{14}$ for ABS composed of PEG-600 $+[\mathrm{Ch}] \mathrm{Cl}+\mathrm{H}_{2} \mathrm{O}(\varpi)$ and PEG$600+[\mathrm{Ch}][\mathrm{OAc}]+\mathrm{H}_{2} \mathrm{O}(\bullet)$ at $25^{\circ} \mathrm{C}$ and seven mixture points $(\bullet)$ used in the liquid-liquid extraction of 
caffeine.

As shown in Figure 1, for each type of ABS two different screenings were performed, a first one to investigate how the PEG- 600 concentration affects the caffeine partitioning (points 1 to 4 ), and a second one where it was evaluated how the caffeine partition changes with the salt concentration (points 4 to 6). The mixture point 7 (56 $\mathrm{wt} \%$ of PEG-600 $+23 \mathrm{wt} \%$ of Salt/IL) was chosen to validate the caffeine partitioning with the simultaneous decrease of salt and polymer concentration in the system. The partition coefficients of caffeine obtained in the ABS studied are depicted in Figure 2. All the assays were performed in triplicate and the detailed average and standard deviations of the weight fraction compositions (wt\%), partition coefficients $(\mathrm{K})$, volume and weight ratios $\left(\mathrm{R}_{\mathrm{V}}\right.$ and $\left.\mathrm{R}_{\mathrm{W}}\right)$ for each ABS are provided in Table S1 in the Supporting Information.

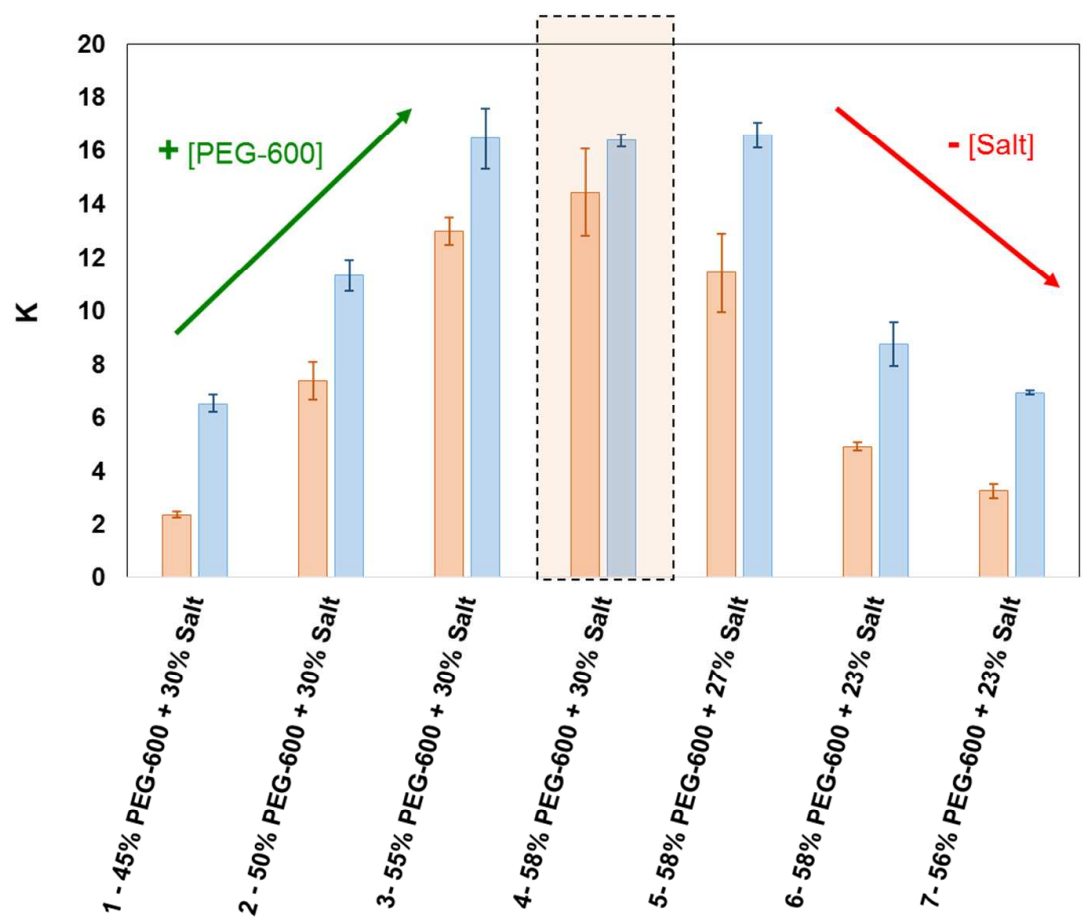

Figure 2. Caffeine partition coefficients (K) in ABS composed of PEG-600 $+[\mathrm{Ch}] \mathrm{Cl}+\mathrm{H}_{2} \mathrm{O}(\square)$ and PEG-600 $+[\mathrm{Ch}][\mathrm{OAc}]+\mathrm{H}_{2} \mathrm{O}(\square)$ at $25^{\circ} \mathrm{C}$. The error bars correspond to the standard deviation of three independent assays.

The results obtained reveal that caffeine always preferentially partitions to the PEG-rich phase, and that the partition coefficient increases with both the concentration of PEG-600 and salt/IL. In both series of ABS, the highest K was obtained in the system 
composed of $58 \mathrm{wt} \%$ of PEG-600 and $30 \mathrm{wt} \%$ of salt/IL (system 4). From Figure 2 it is also possible to evaluate the effect of the cholinium anion on the partitioning of the alkaloid. At the same composition, the ternary systems with [Ch][OAc] always favour the partition of caffeine towards the PEG-rich phase. It is important to note that the partitioning coefficient of caffeine is independent of the alkaloid concentration in the system in a wide range of concentrations studied as shown in Figure S1 (presented in the Supported Information).

In a previous work we studied the ability of different IL/PEG-2000 based ABS to promote a selective separation of three alkaloids, including caffeine. ${ }^{22}$ It was shown that imidazolium-based ILs with a more hydrophilic nature lead to a preferential partition of caffeine into the IL-rich phase, while more hydrophobic ILs induced an inversion of the caffeine partition to the PEG-rich phase. Although, those partitioning studies were performed at different temperatures than those here investigated (i.e., temperatures higher than $50^{\circ} \mathrm{C}$ ), and with different compounds (i.e., polymers with a higher molecular weight and ILs with significant structural differences), the data obtained suggests that the partitioning of solutes in PEG-IL based ABS is related with the amount of water present in the coexisting phases. Following these previous considerations, herein we measured the water content in the PEG- and salt-rich phases for each ternary mixture (the methodology for the water quantification is detailed in the Experimental Section).

A correlation between the water content in the PEG-rich phase over the salt-rich phase (in weight fraction), defined as $\mathrm{H}_{2} \mathrm{O}$ ratio, and the logarithm of the caffeine partition coefficient $(\operatorname{logK})$ is presented in Figure 3 (detailed data of water content in both PEG and salt-rich phases for each ternary system are presented in the Supporting Information, Table S1). 


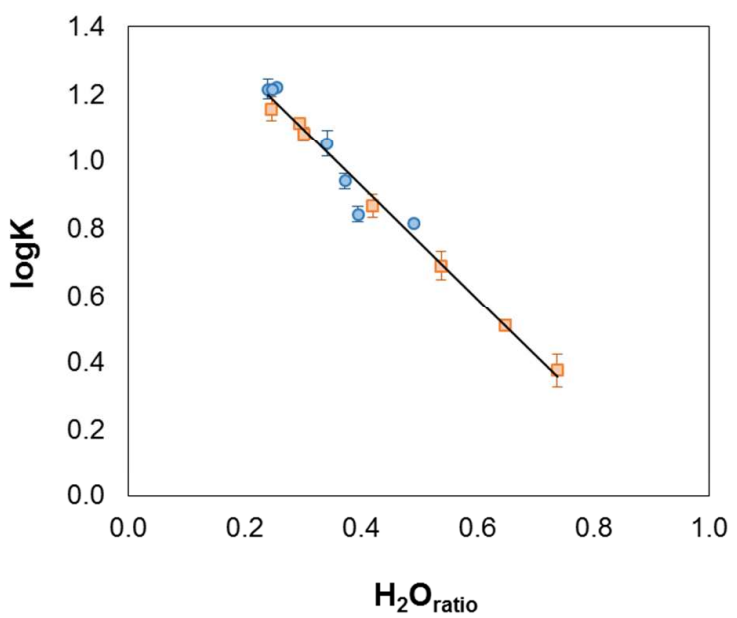

Figure 3. Logarithmic function of the caffeine partition coefficients $(\log \mathrm{K})$ as a function of $\mathrm{H}_{2} \mathrm{O}$ ratio in the ABS composed of PEG-600 $+[\mathrm{Ch}] \mathrm{Cl}+\mathrm{H}_{2} \mathrm{O}(\square)$ and PEG- $600+[\mathrm{Ch}][\mathrm{OAc}]+\mathrm{H}_{2} \mathrm{O}(\bullet)$ at $25^{\circ} \mathrm{C}$. The black line corresponds to the linear function. The error bars correspond to the standard deviation of three independent assays.

The linear dependency obtained in Figure 3 supports a clear dependence of the solute partition on the water present in each phase. Thus, independently of the salt used, an increase of the water content in the PEG-rich phase decreases the caffeine partition coefficient. In general, it is evident that the strong hydrophobic character of the PEGrich phase favours the migration of caffeine into it, being this influence strengthened with the decrease of the water distribution between the polymer- and salt-rich phases.

Although the relationship between the solute partition the and water content could provide novel evidences on the mechanisms driving the partition of hydrophobic or mildly hydrophilic solutes in $[\mathrm{Ch}]^{+}$-salts/PEG ABS, it is important to carefully analyse the type of compounds present in each system. As shown in Figure 2, $[\mathrm{Ch}][\mathrm{OAc}]$ always leads to higher $\mathrm{K}$ values than $[\mathrm{Ch}] \mathrm{Cl}$. The data for the water content of the salt-rich phase presented in Supporting Information, Table S1, show that in ABS composed of $[\mathrm{Ch}][\mathrm{OAc}]$ the water concentration in the salt-rich phase is always higher than in the systems with $[\mathrm{Ch}] \mathrm{Cl}$. As expected, the higher basicity of the acetate anion ${ }^{23}$, will induce a preferential solvation of the anion and, consequently, will dehydrate more the polymer-rich phase, than the Cl-based salt.

Overall, the caffeine partition coefficients obtained in the studied PEG/[Ch $]^{+}-$ ABS show that the separation of solutes is mainly a matter of solute-water "nonaffinity", in which hydrophobic solutes will concentrate in the phases with lower water content, while hydrophilic solutes will partition preferentially towards the salt/IL-rich 
phase. This trend is further supported hereafter by studying the partition of a series of alkaloids of variable polarity.

\section{Partitioning of alkaloids in PEG-600/[Ch]Cl ABS}

To further confirm the hypothesis previously suggested, the partitioning of two other xanthine-based alkaloids, theobromine and theophylline, in PEG- $600 /[\mathrm{Ch}] \mathrm{Cl}$ was investigated. As caffeine, these alkaloids are also included in the group of methylxanthines - heterocyclic organic compounds with pyrimidinedione and imidazole rings. ${ }^{24}$ Table 1 presents a comparison of their chemical structure, $\log \mathrm{K}_{\mathrm{ow}}$, and constant of acidic dissociation $\left(\mathrm{pK}_{\mathrm{a}}\right)$.

Table 1. Chemical structure of the studied alkaloids, $\log \mathrm{K}_{\mathrm{ow}}$ and $\mathrm{pKa}$.

\begin{tabular}{clll}
\hline Name & $\mathbf{L o g K}_{\mathbf{0 w}}$ & $\mathbf{p K}_{\mathbf{a} 1} / \mathbf{p K}_{\mathbf{a 2}}$ \\
\hline Chemical Structure & $0.12 / 10.5^{25}$ \\
Theophylline & $-0.77^{21}$ & $0.6 / 14^{25}$ \\
\hline
\end{tabular}

As can be inferred from Table 1, the three alkaloids differ in the number of methyl groups attached to the aromatic cores. Compared to the simplest xanthine molecule, the addition of two methyl groups forms the theophylline and the theobromine structures whereas the addition of three methyl groups leads to the caffeine molecule. These changes on the molecular structures lead to different affinities for water, as can be evaluated by the $\log \mathrm{K}_{\mathrm{ow}}$ values presented in Table 1, which follow the increasing hydrophobicity trend: theobromine $>$ caffeine $>$ theophylline. According to this trend, we expect theobromine to exhibits the lowest aptitude to partition for the PEG-rich phase; however, since its $\log \mathrm{K}_{\mathrm{ow}}$ is lower than the ABS phase-forming agents, it may, on the other hand, concentrate preferentially in the more organic phase (polymer-rich phase). 
The partition coefficients of the three alkaloids were determined in three mixture compositions for the ABS composed of [Ch]Cl (at circa 30, 27 and $23 \mathrm{wt} \%$ ) and PEG600 (at circa $58 \mathrm{wt} \%$ ). As stated before, due to their different water affinities, these methylxanthines can be a useful tool to evaluate the relative hydrophobicities of the coexisting phases of $\mathrm{PEG} /[\mathrm{Ch}]^{+}$-salts ABS. Accordingly, the respective logarithmic function of the partition coefficient of each alkaloid $(\log K)$ should be related with $\log \mathrm{K}_{\mathrm{ow}}$ in each ternary mixture, as can be gauged from Figure 4.
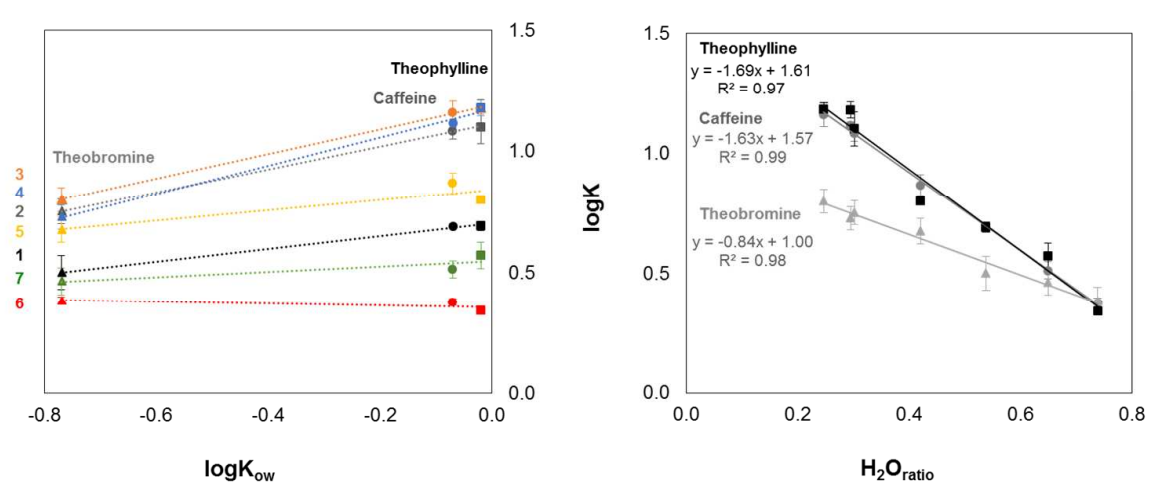

Figure 4. (Left) - Linear relationship between the $\log \mathrm{K}$ versus $\log \mathrm{K}_{\mathrm{ow}}$ at different ternary phase compositions (mixture points 1 to 7 reported in Table S1 in the Supporting Information). (Right) - Linear relationship between $\log \mathrm{K}$ as a function of $\mathrm{H}_{2} \mathrm{O}$ ratio. The error bars correspond to the standard deviation of three independent assays.

The results depicted in Figure 4 (left) show that as caffeine, theobromine and theophylline also preferentially partition to the PEG-rich phase $(\log \mathrm{K}>0)$. In addition, in most of the PEG-600/[Ch]Cl systems, it was observed the same partitioning trend: theobromine $<$ caffeine $<$ theophylline. As expected, the partition of the alkaloids to the PEG-rich phase follows their lipophilic character (Table 1), in which an increase of the alkaloid hydrophobicity (reflected by the increase of the $\mathrm{K}_{\mathrm{ow}}$ value) increases its affinity for the most hydrophobic phase (PEG-rich phase). Moreover, as observed in Figure 4 (right), and as demonstrated previously for caffeine, both theobromine and theophylline partition coefficients (logarithmic functions) follow a linear trend with the water distribution between the PEG-rich and salt-rich phases. As the lipophilicity of theophylline and caffeine are very similar (-0.07 and -0.02 , respectively) their dependence on the $\mathrm{H}_{2} \mathrm{O}$ ratio is almost the same, whereas theobromine shows a decrease on its partition as a function of the water distribution.

In summary, the alkaloids partition coefficients are directly related with the 
hydrophilicity/hydrophobicity of each solute and with the relative hydrophilicity of the phases in equilibrium for the $\mathrm{PEG} /[\mathrm{Ch}] \mathrm{Cl} \mathrm{ABS}$. As discussed above for caffeine, although the two phases of ABS are rich in water, the less hydrophilic environment (low $\mathrm{H}_{2} \mathrm{O}$ ratio between the PEG- and salt-rich phase) of the polymer-rich phase leads to an increase on the solute-polymer hydrophobic interactions, and consequently, to an increase on the partition of alkaloids for the PEG-rich phase.

The partition coefficients of the studied alkaloids suggest that the solutes partitioning on ABS composed of PEG-600 and [Ch] $]^{+}$-based salts results mainly from their water affinity, or, in this case, non-affinity. However, a number of additional questions still remain: Is this dependency valid for other types of PEG-600-based ABS composed of other $[\mathrm{Ch}]^{+}$-based salts? How does it work if, for example, we perform the separation of theophylline in a system where the $\mathrm{pH}$ of the phases in equilibrium is above their $\mathrm{pK}_{\mathrm{a} 2}$ (8.6)? Is this dependency valid for other solutes?

In order to validate and confirm the main findings derived from the experimental results, while considering in more detail the chemical nature of the alkaloids under consideration, COSMO-RS was further applied to compute the screening charge distributions on the molecular surface (the so-called $\sigma$-profile), aiming at qualitatively describe the lipophilicity, hydrophilicity and hydrogen bonding capacity of each alkaloid. COSMO-RS is the quantum-chemistry based method developed by Klamt and coworkers $^{26,27}$ to predict the thermodynamic properties of fluid mixtures based on the chemical potentials of each species in solution, $\mu$, which are calculated from the screening charge densities, $\sigma$, on the surface of molecules. The COSMO-RS $\sigma$-profile histogram can be divided into three main regions upon the following cut-off values: the hydrogen bond (HB) donor region $\left(\sigma<-0.0082 \mathrm{e} / \AA^{2}\right)$, the HB acceptor region $(\sigma>$ $\left.+0.0082 \mathrm{e} / \AA^{2}\right)$ and the non-polar region $\left(-0.0082<\sigma<+0.0082 \mathrm{e} / \AA^{2}\right)$. Figure 5a) shows the $\sigma$-profile of the three alkaloids (caffeine, theobromine and theophylline). The presence of a prominent peak with a broad charge distribution in the non-polar region of the $\sigma$-profiles of the alkaloids indicates their remarkable lipophilic character; such feature illustrates the chemical behavior of the alkaloids and supports their preferential partition for the PEG-rich phase in the ABS experimentally studied. However, the charge distribution around the $\sigma \sim 0.0015 \mathrm{e} / \AA^{2}$ region on the $\sigma$-profile of the alkaloids indicates their ability to act as a HB acceptor through the electron pairs of oxygen and nitrogen atoms, which may promote their interaction with water and the $[\mathrm{Ch}]^{+}$cation, since the latter presents an acidic behaviour as depicted in the $\sigma$-profiles for the salts 
presented in Figure 5b).

a)

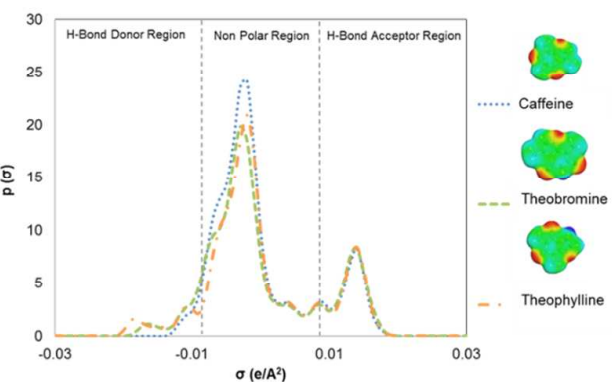

b)

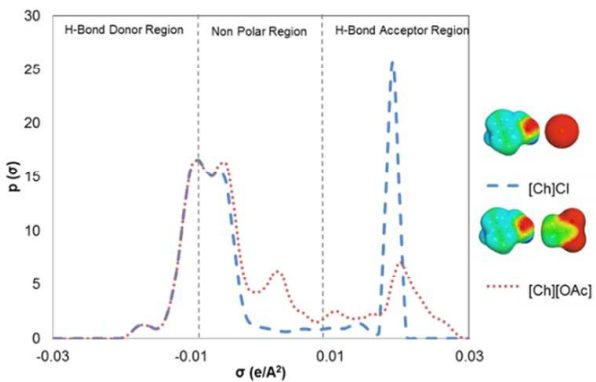

Figure 5. Representation of $\sigma$-profiles for alkaloids (a) and $[\mathrm{Ch}]^{+}$-salts (b).

According to Figure 5, there is a slight distribution of charge around the HB donor region of the $\sigma$-profiles for the alkaloids, mainly due to the $\mathrm{H}$ atoms attached to electronegative atoms, such as nitrogen in the case of theobromine and theophylline (the peak is lower for caffeine due to the substitution of the $\mathrm{H}$ atom for a methyl group). This fact may promote HB bond interactions with more basic anions like [OAc]', which presents a strong HB acceptor ability as depicted by the broad distribution of charge present at the positive $\sigma$ region of the $\sigma$-profile. However, it should be noted that HB acceptor anions such as $[\mathrm{OAc}]^{-}$may promote anion-cation HB interactions with the $[\mathrm{Ch}]^{+}$cation, which could compete with the interactions between other solutes and the ILs, as well as enhance the interactions between the IL and water.

\section{Anion influence in the partitioning of alkaloids in PEG-600/[Ch $]^{+}$-salts ABS}

The analysis of the solutes partition coefficients in ABS composed of PEG-600 and $[\mathrm{Ch}][\mathrm{OAc}]$ or $[\mathrm{Ch}] \mathrm{Cl}$ revealed that the water content of the phases plays a primordial role on their partition. Therefore, aiming at evaluating the anion influence in the separation of each methylxanthine, other three PEG-600/[Ch $]^{+}$-salt systems were additionally studied ([Ch][Bic], $[\mathrm{Ch}][\mathrm{DHcit}]$ and $[\mathrm{Ch}][\mathrm{DHph}]$ ). The partition coefficients of caffeine, theobromine and theophylline were compared with previous data obtained for the systems with $[\mathrm{Ch}][\mathrm{OAc}]$ and $[\mathrm{Ch}] \mathrm{Cl}$. Since the $[\mathrm{Ch}]^{+}$-salts are synthesized with different acids, the $\mathrm{pH}$ of the system at equilibrium will also change. Thus, as further information, the $\mathrm{pH}$ values of both phases (PEG-600-rich and salt-rich phases) were collected and compared with the partition coefficient of each alkaloid for the ABS studied (Figure 6). The detailed data, weight fractions compositions, alkaloids' partition coefficients and $\mathrm{pH}$ of the coexisting phases are represented in Table $\mathrm{S} 2$ in the 
Supporting Information.

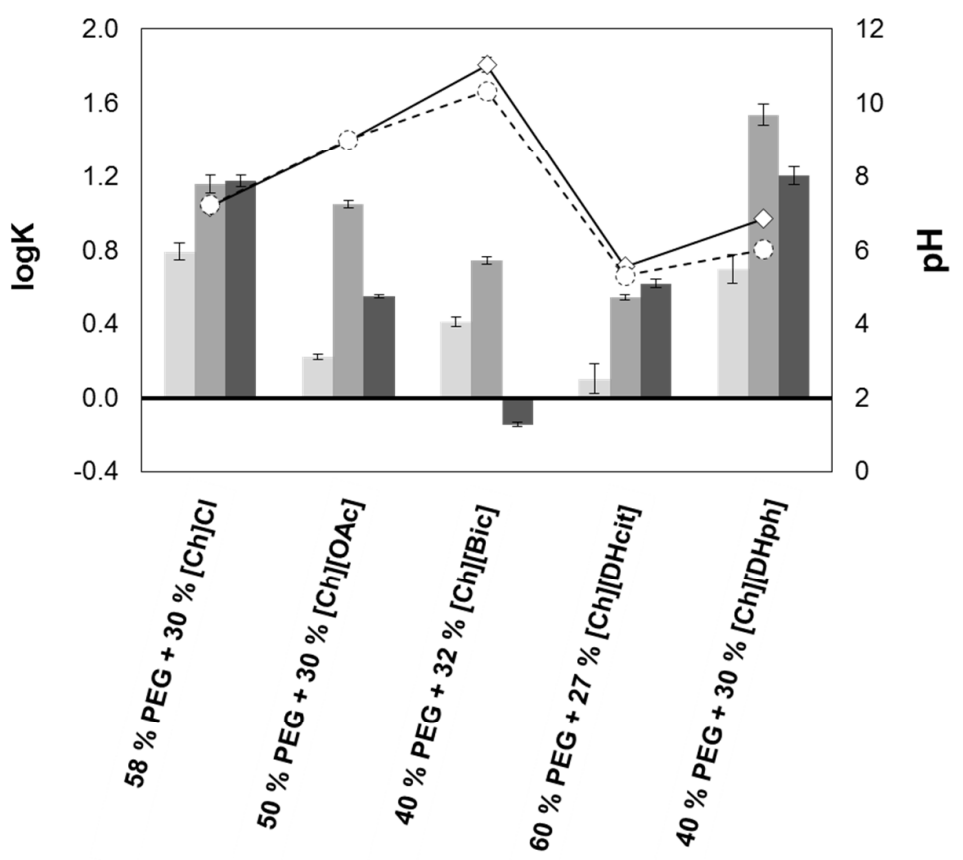

Figure 6. Logarithmic function of the caffeine partition coefficients $(\operatorname{logK})$ of theobromine $(\square)$, caffeine ( ) and theophylline ( ), and $\mathrm{pH}$ values of both PEG-rich $(\diamond)$ and salt-rich phases $(\circ)$ in the systems composed of different $[\mathrm{Ch}]^{+}$-based salts and PEG- 600 at $25^{\circ} \mathrm{C}$. The error bars correspond to the standard deviation of three independent assays.

In general, all alkaloids preferentially partition to the PEG-rich phase independently of the composition and $[\mathrm{Ch}]^{+}$-salt employed in the ABS formulation. However, one exception was observed with theophylline for the system composed of $[\mathrm{Ch}][\mathrm{Bic}]$. In this system, the biomolecule is more concentrated in the salt-rich phase in contrast with the remaining alkaloids. Since the $\mathrm{pK}_{\mathrm{a} 2}$ of theophylline (8.6) is inferior to the $\mathrm{pH}$ of the phases $(10.33 \pm 0.01$ and $11.03 \pm 0.19)$, theophylline is negatively charged (alkaloids' speciation curves are depicted in Figure S2 in the Supporting Information). Therefore, in this particular example, electrostatic interactions between the charged solute and the salt ions can explain this inversion on the migration pattern. The proximity of the $\mathrm{pH}$ of the phases of the acetate-based system (around 9) with the dissociation constant of theophylline also explains the decrease in its partition coefficient (in comparison with caffeine). In this system, theophylline exists as an approximately equimolar mixture of charged and non-charged species. For the same reason, the presence of charged theophylline decreases the partition coefficient due to a preferential partition for the salt-rich phase.

Previously we demonstrated for the PEG-600/[Ch]Cl a partitioning trend closely 
related with the lipophilicity $\left(\log \mathrm{K}_{\mathrm{ow}}\right)$ of the solutes. As observed in Figure 6, however, only $[\mathrm{Ch}][\mathrm{DHcit}]$ follows a similar tendency on the methylxanthines partition for the PEG-rich phase (theobromine $<$ caffeine $<$ theophylline) as defined by their lipophilicity gradient. Nonetheless, it is important to note that only in this system the $\mathrm{pH}$ is low enough to avoid the presence of charged xanthines, in particular for theobromine and theophylline. Taking into account that caffeine is the only non-charged species in the $\mathrm{pH}$ range achieved in the diverse systems, it can be used as a probe of hydrophobicity. Additionally, the odd trend observed with $[\mathrm{Ch}][\mathrm{DHph}]$ could be a direct result of specific interactions occurring between the solute and the [DHph $]^{-}$anion.

To better understand the odd trend observed with [Ch][DHph], and to further evaluate the affinity of the salt anion for each alkaloid, COSMO-RS was applied to evaluate the excess enthalpy of mixing and the activity coefficients at infinite dilution as essential thermodynamic properties. Previously, it was proved COSMO-RS to be capable to reproduce the experimental excess enthalpies of systems containing ILs. ${ }^{28}$ In addition, previous studies demonstrated the relationship between the activity coefficients of different solutes in ILs and the excess enthalpies of those mixtures. ${ }^{29}$ Therefore, in the light of the aforementioned results, herein we aim at analyse the activity coefficients and the excess enthalpies between the alkaloids solutes and three $[\mathrm{Ch}]^{+}$-salts, namely $[\mathrm{Ch}][\mathrm{DHph}],[\mathrm{Ch}][\mathrm{Cl}]$ and $[\mathrm{Ch}][\mathrm{OAc}]$, in order to acquire further insights into the different behaviour of the systems (Figure 7). Excess enthalpies and energetic contributions for the alkaloid-salts mixtures calculated by COSMO-RS over the whole range of concentrations are presented in Figure S3 in the Supporting Information. There, it can be seen that equimolar concentrations of the systems represent a good approach to illustrate the intermolecular interactions within the mixture, and hence they were deemed appropriate to proceed with the subsequent analyses. 

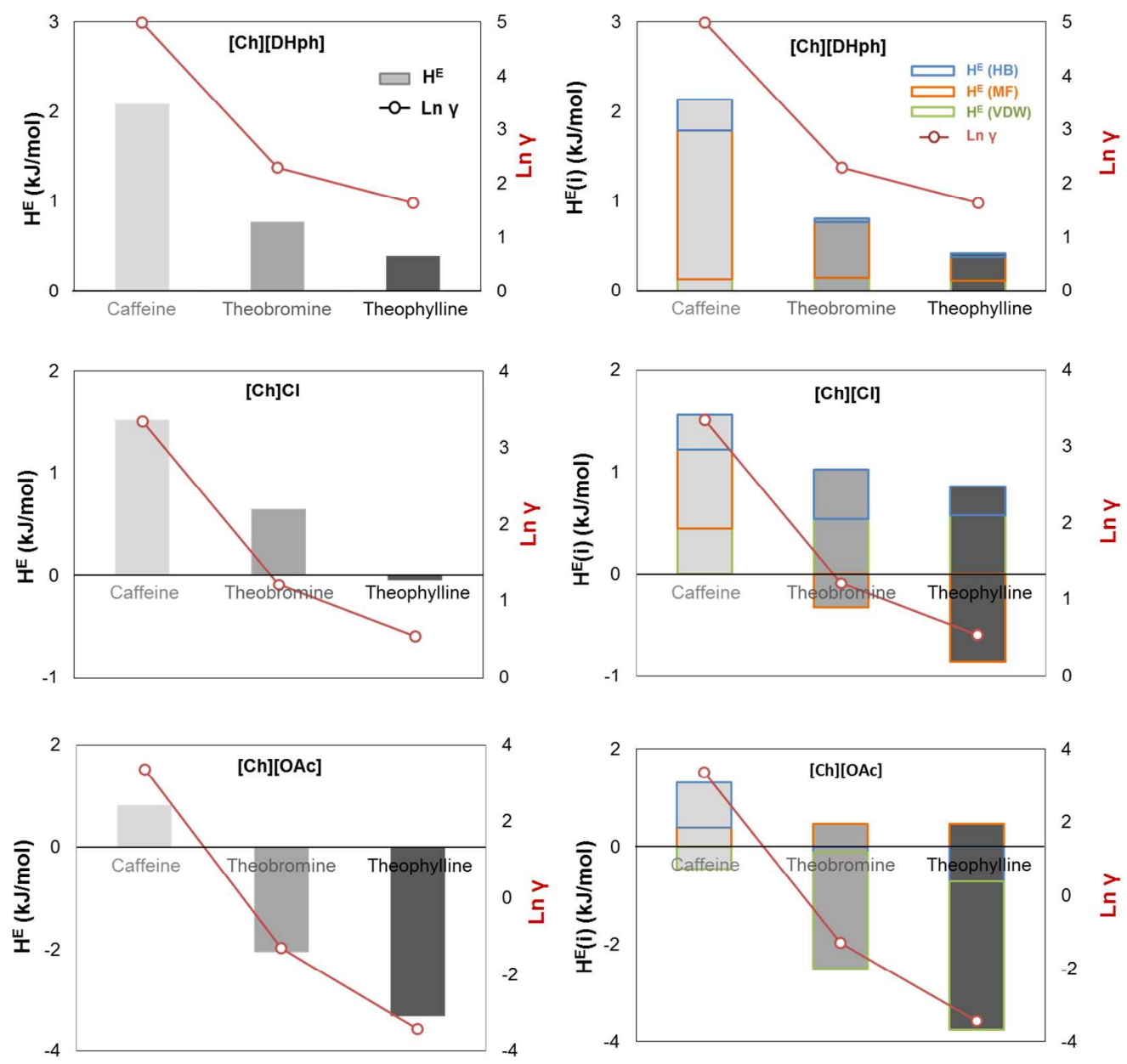

Figure 7. Excess enthalpy, $\mathrm{H}^{\mathrm{E}}$, and energetic contributions, $\mathrm{H}(\mathrm{i})$, versus activity coefficient, $\operatorname{Ln}(\gamma)$, of alkaloids in $[\mathrm{Ch}]^{+}$-salts computed by COSMO-RS at $25^{\circ} \mathrm{C}$.

Figure 7 compares the activity coefficients of the alkaloids in each salt combined with the excess enthalpy of the systems. Overall, both the activity coefficients and the excess enthalpies of the alkaloids in the $[\mathrm{Ch}]^{+}$-salts decrease from $[\mathrm{Ch}][\mathrm{DHph}]$ to $[\mathrm{Ch}][\mathrm{Cl}]$ to $[\mathrm{Ch}][\mathrm{OAc}]$, which means an increasing affinity between the solutes and the anions in the following order: $[\mathrm{DHph}]^{-}<[\mathrm{Cl}]^{-}<$[OAc $^{-}$(note that decreasing activity coefficients means increasing interactions between the solute and the solvent, and that negative -exothermic- excess enthalpies involve increasing attractive interactions within the components of the mixture). In addition, it can be noted that alkaloids presenting $\ln (\gamma)<0$ in the $[\mathrm{Ch}]^{+}$-salts (attractive forces dominate) always present exothermic excess enthalpies of mixing (i.e., stronger interactions between unlike species compared to like species), hence supporting the premise that the relationship between the activity coefficients and the excess enthalpies seems a sensible approach to analyse the behaviour of the systems. 
Subsequently, COSMO-RS was applied to compute the detailed energetic contributions to the excess enthalpies of mixtures - van der Waals, H(VDW); hydrogen bonding, $\mathrm{H}(\mathrm{HB})$; and electrostatics-misfits, $\mathrm{H}(\mathrm{MF})$ - to gain a deeper insight into the molecular interactions occurring within the studied systems. Alkaloids do not present affinity towards the $[\mathrm{Ch}][\mathrm{DHph}]$ salt, mainly due to the repulsive electrostatic interactions between the solute and the $[\mathrm{DHph}]^{-}$anion, which is a bulky anion with disperse charge, which explains the increased alkaloids partitioning for the PEG-600 rich phase. Such repulsive electrostatic interactions decrease at some extent when pairing the $[\mathrm{Ch}]^{+}$cation with the $[\mathrm{Cl}]^{-}$anion; however, the repulsive van der Waals interactions between the halogenated anion and the alkaloids still promote a low affinity between the solute and the solvents. Lastly, $[\mathrm{Ch}]^{+}$-salts paired with basic anions like [OAc] notably increase the affinity towards the alkaloids, in particular theobromine and theophylline, through hydrogen bonding, which is in agreement with the lower partition towards the PEG-rich phase experimentally observed for these two alkaloids in ABS. These results show that, although the water ratio content between the phases can be an important driving force for the partition, as shown above in Figure 3, other factors as direct interactions between the solute and the phase-forming compounds also play an important role, that mat explain the differences observed for the various alkaloids shown in Figure 4.

In general, both experimental and computational results demonstrate that the non-charged solutes (as caffeine) could be used as an effective and valuable probe to characterize the relative hydrophobicity of a particular series of ABS, particularly when hydrophobic type interactions are predominant. Some specific exceptions, as observed in $[\mathrm{Ch}][\mathrm{DHph}] / \mathrm{PEG}-600$ based ABS, may occur, in which other type of interactions are predominant (specifically, repulsive electrostatic interactions).

Recently, we demonstrated that different types of imidazolium-based ILs are able to promote a selective separation of caffeine when combined with an aqueous solution of PEG-2000. ${ }^{22}$ In most systems studied, caffeine preferentially partitioned into the PEG-rich phase, as observed here. Other types of IL-based ABS used on studies of the extraction or separation of caffeine were formed by ILs/salts ${ }^{30,31}$ and ILs/carbohydrates. ${ }^{6}$ In all these types of ABS, the caffeine partitions preferentially to the IL-rich phase. In general, all previous results with IL-based ABS suggest that the caffeine distribution is effectively dependent on the relative hydrophobic balance between the coexisting phases. Thus, using caffeine as a hydrophobicity probe through 
the respective partitioning values it is possible to define the more "hydrophobic" phase in each type of IL-based ABS as according to:

- a) IL or salt/PEG ABS - PEG-rich phase;

- b) IL/salt ABS - IL-rich phase;

- c) IL/carbohydrate ABS - IL-rich phase.

As final remarks, the experimental results on the partition coefficients of alkaloids indicate that at in all the studied conditions these solutes preferentially partition to the more hydrophobic PEG-rich phase. Furthermore, it was demonstrated a linear dependence between its distribution between the coexisting phases and the water ratio between the two phases. Nevertheless, in particular cases, specific interactions (such as repulsive electrostatic interactions) between each $[\mathrm{Ch}]^{+}$-salt and alkaloid, as well as the presence of charged and non-charged species in solution, cannot be discarded and may change the partition trend behaviour.

It was here demonstrated that the caffeine partitioning can be used to evaluate the relative hydrophobicity of the phases in IL-based ABS, making of it a powerful tool to characterize ABS. Moreover, the understanding of hydrophobic/hydrophilic balance of ABS can further help on the development of predictive models for the solutes' separation, supporting a future application of ABS as an effective platform for the separation of high-value biomolecules at industrial scale.

\section{Supporting Information}

The document contains information related to the detailed experimental data of all the ternary systems studied (weigh fraction compositions; tie-lines; tie-line lengths; partition coefficients of caffeine; theobromine and theophylline; volume ratios; weight ratio; water content; $\mathrm{pH}$, conductivity) and alkaloids' speciation curves as function of $\mathrm{pH}$. The document contains also the detailed description of the standard procedure for COSMO_RS predictions and the excess enthalpies, $\mathrm{H}^{\mathrm{E}}$, and detailed energetic contributions, $\mathrm{H}(\mathrm{i})$, of alkaloids in $[\mathrm{Ch}]^{+}$-based salts computed by COSMO-RS at $25^{\circ} \mathrm{C}$.

\section{Acknowledgements}

This work was developed in the scope of two projects, namely: young investigator FAPESP (Ref. 2014/16424-7) financed by FAPESP (Sao Paulo Research Foundation Brazil); and project Sprint-2015 University of Manchester/FAPESP (Ref. 2015/50058- 
$0)$ co-financed by FAPESP and University of Manchester. This work was also developed in the scope of the project CICECO-Aveiro Institute of Materials (Ref. FCT UID/CTM/50011/2013), financed by national funds through the FCT/MEC and FEDER under the PT2020 Partnership Agreement. The authors also acknowledge FCT for the doctoral grant SFRH/BD/100155/2014 of M.V. Quental. M.G. Freire acknowledges the European Research Council (ERC) for the Starting Grant ERC-2013-StG-337753. 


\section{References}

(1) Hatti-Kaul, R. Aqueous two-phase systems. Mol. Biotechnol. 2001, 19 (3), 269277.

(2) Zaslavsky, B. Y. Aqueous Two-Phase Partitioning: Physical Chemistry and Bioanalytical Applications; Taylor \& Francis, 1994.

(3) Gutowski, K. E.; Broker, G. A.; Willauer, H. D.; Huddleston, J. G.; Swatloski, R. P.; Holbrey, J. D.; Rogers, R. D. Controlling the aqueous miscibility of ionic liquids: Aqueous biphasic systems of water-miscible ionic liquids and waterstructuring salts for recycle, metathesis, and separations. J. Am. Chem. Soc. 2003, 125 (22), 6632-6633.

(4) Freire, M. G.; Cláudio, A. F. M.; Araújo, J. M. M.; Coutinho, J. A. P.; Marrucho, I. M.; Lopes, J. N. C.; Rebelo, L. P. N. Aqueous biphasic systems: a boost brought about by using ionic liquids. Chem. Soc. Rev. 2012, 41 (14), 4966.

(5) Pereira, J. F. B.; Rebelo, L. P. N.; Rogers, R. D.; Coutinho, J. A. P.; Freire, M. G. Combining ionic liquids and polythylene glycols to boost the hydrophobichydrophilic range of aqueous biphasic systems. Phys. Chem. Chem. Phys. 2013, 15 (45), 19580-19583.

(6) Freire, M. G.; Louros, C. L. S.; Rebelo, L. P. N.; Coutinho, J. A. P. Aqueous biphasic systems composed of a water-stable ionic liquid + carbohydrates and their applications. Green Chem. 2011, 13 (6), 1536.

(7) Soares, R. R. G.; Azevedo, A. M.; Van Alstine, J. M.; Aires-Barros, M. R. Partitioning in aqueous two-phase systems: Analysis of strengths, weaknesses, opportunities and threats. Biotechnol. J. 2015, 10 (8), 1158-1169.

(8) Cabral, J. M. S. Cell partitioning in aqueous two-phase polymer systems. $A d v$ Biochem. Eng. Biotechnol. 2007, 106, 151-171.

(9) Rogers, R. D.; Seddon, K. R. Chemistry. Ionic liquids-solvents of the future? Science 2003, 302 (5646), 792-793.

(10) Petkovic, M.; Seddon, K. R.; Rebelo, L. P. N.; Silva Pereira, C. Ionic liquids: a pathway to environmental acceptability. Chem. Soc. Rev. 2011, 40 (3), 13831403.

(11) Anastas, P.; Eghbali, N. Green chemistry: principles and practice. Chem. Soc. Rev. 2010, 39 (1), 301-312.

(12) Pernak, J.; Syguda, A.; Mirska, I.; Pernak, A.; Nawrot, J.; Prạdzyńska, A.; Griffin, S. T.; Rogers, R. D. Choline-derivative-based ionic liquids. Chem. - A Eur. J. 2007, 13 (24), 6817-6827. 
(13) Petkovic, M.; Ferguson, J. L.; Gunaratne, H. Q. N.; Ferreira, R.; Leitão, M. C.; Seddon, K. R.; Rebelo, L. P. N.; Pereira, C. S. Novel biocompatible choliniumbased ionic liquids - toxicity and biodegradability. Green Chem. 2010, 30 (4), 643-649.

(14) Pereira, J. F. B.; Kurnia, K. A.; Cojocaru, O. A.; Gurau, G.; Rebelo, L. P. N.; Rogers, R. D.; Freire, M. G.; Coutinho, J. A. P. Molecular interactions in aqueous biphasic systems composed of polyethylene glycol and crystalline vs. liquid cholinium-based salts. Phys. Chem. Chem. Phys. 2014, 16 (12), 5723-5731.

(15) Mourão, T.; Tomé, L. C.; Florindo, C.; Rebelo, L. P. N.; Marrucho, I. M. Understanding the Role of Cholinium Carboxylate Ionic Liquids in PEG-Based Aqueous Biphasic Systems. ACS Sustain. Chem. Eng. 2014, 2 (10), 2426-2434.

(16) Liu, X.; Li, Z.; Pei, Y.; Wang, H.; Wang, J. (Liquid + liquid) equilibria for (cholinium-based ionic liquids + polymers) aqueous two-phase systems. J. Chem. Thermodynamics 2013, 60, 1-8.

(17) Tomé, L. I. N.; Pereira, J. F. B.; Rogers, R. D.; Freire, M. G.; Gomes, J. R. B.; Coutinho, J. A. P. "Washing-out" ionic liquids from polyethylene glycol to form aqueous biphasic systems. Phys. Chem. Chem. Phys. 2014, 16 (6), 2271-2274.

(18) Pereira, J. F. B.; Kurnia, K. A.; Freire, M. G.; Coutinho, J. A. P.; Rogers, R. D. Controlling the Formation of Ionic-Liquid-based Aqueous Biphasic Systems by Changing the Hydrogen-Bonding Ability of Polyethylene Glycol End Groups. ChemPhysChem 2015, 16 (10), 2219-2225.

(19) Li, Z.; Liu, X.; Pei, Y.; Wang, J.; He, M. Design of environmentally friendly ionic liquid aqueous two-phase systems for the efficient and high activity extraction of proteins. Green Chem. 2012, 14 (10), 2941-2950.

(20) Bezold, F.; Goll, J.; Minceva, M. Study of the applicability of non-conventional aqueous two-phase systems in counter-current and centrifugal partition chromatography. J. Chromatogr. A 2015, 1388, 126-132.

(21) Machatha, S. G.; Yalkowsky, S. H. Comparison of the octanol/water partition coefficients calculated by $\mathrm{C} \log \mathrm{P} \AA, \mathrm{ACD} \log \mathrm{P}$ and KowWin ${ }^{\circledR}$ to experimentally determined values. Int. J. Pharm. 2005, 294 (1-2), 185-192.

(22) Pereira, J. F. B.; Ventura, S. P. M.; e Silva, F. A.; Shahriari, S.; Freire, M. G.; Coutinho, J. A. P. Aqueous biphasic systems composed of ionic liquids and polymers: A platform for the purification of biomolecules. Sep. Purif. Technol. 2013, 113, 83-89.

(23) Cláudio, A. F. M.; Swift, L.; Hallett, J. P.; Welton, T.; Coutinho, J. A. P.; Freire, M. G. Extended scale for the hydrogen-bond basicity of ionic liquids. Phys. Chem. Chem. Phys. 2014, 16 (14), 6593-6601. 
(24) Talik, P.; Krzek, J.; Ekiert, R. J. Analytical Techniques Used for Determination of Methylxanthines and their Analogues-Recent Advances. Sep. Purif. Rev. 2012, 41 (1), 1-61.

(25) Perez-Martinez, I.; Sagrado, S.; Medina-Hernandez, M. J. A rapid procedure for the determination of caffeine, theophylline and theobromine in urine by micellar liquid chromatography and direct sample injection. Anal Chim Acta, 1995, 304, 195-201.

(26) Eckert, F.; Klamt, A. Fast Solvent Screening via Quantum Chemistry: COSMORS Approach. AIChE J. 2002, 48 (2), 369-385.

(27) Eckert, F. and Klamt, A. COSMOtherm. COSMOlogic GmbH \& Co. , KG,: Leverkusen, Germany. 2014.

(28) Kurnia, K. A.; Coutinho, J. A. P. Overview of the excess enthalpies of the binary mixtures composed of molecular solvents and ionic liquids and their modeling using COSMO-RS. Ind. Eng. Chem. Res. 2013, 52 (38), 13862-13874.

(29) Gonzalez-Miquel, M.; Palomar, J.; Rodriguez, F. Selection of Ionic Liquids for Enhancing the Gas Solubility of Volatile Organic Compounds. J. Phys. Chem. B 2013, 117 (1), 296-306.

(30) Freire, M. G.; Neves, C. M. S. S.; Marrucho, I. M.; Canongia Lopes, J. N.; Rebelo, L. P. N.; Coutinho, J. A. P. High-performance extraction of alkaloids using aqueous two-phase systems with ionic liquids. Green Chem. 2010, 12 (10), 1715-1718.

(31) Louros, C. L. S.; Cláudio, A. F. M.; Neves, C. M. S. S.; Freire, M. G.; Marrucho, I. M.; Pauly, J.; Coutinho, J. A. P. Extraction of biomolecules using phosphonium-based ionic liquids $+\mathrm{K}_{3} \mathrm{PO}_{4}$ aqueous biphasic systems. Int. J. Mol. Sci. 2010, 11 (4), 1777-1791. 


\title{
For Table of Contents Use Only
}

\section{Alkaloids as Alternative Probes to Characterize the Relative Hydrophobicity of Aqueous Biphasic Systems}

\author{
Jorge F. B. Pereira ${ }^{1 *}$, Agnes Magri ${ }^{1}$, Maria V. Quental, ${ }^{2}$ Maria Gonzalez-Miquel ${ }^{3}$, Mara \\ G. Freire ${ }^{2}$, João A. P. Coutinho ${ }^{2}$
}

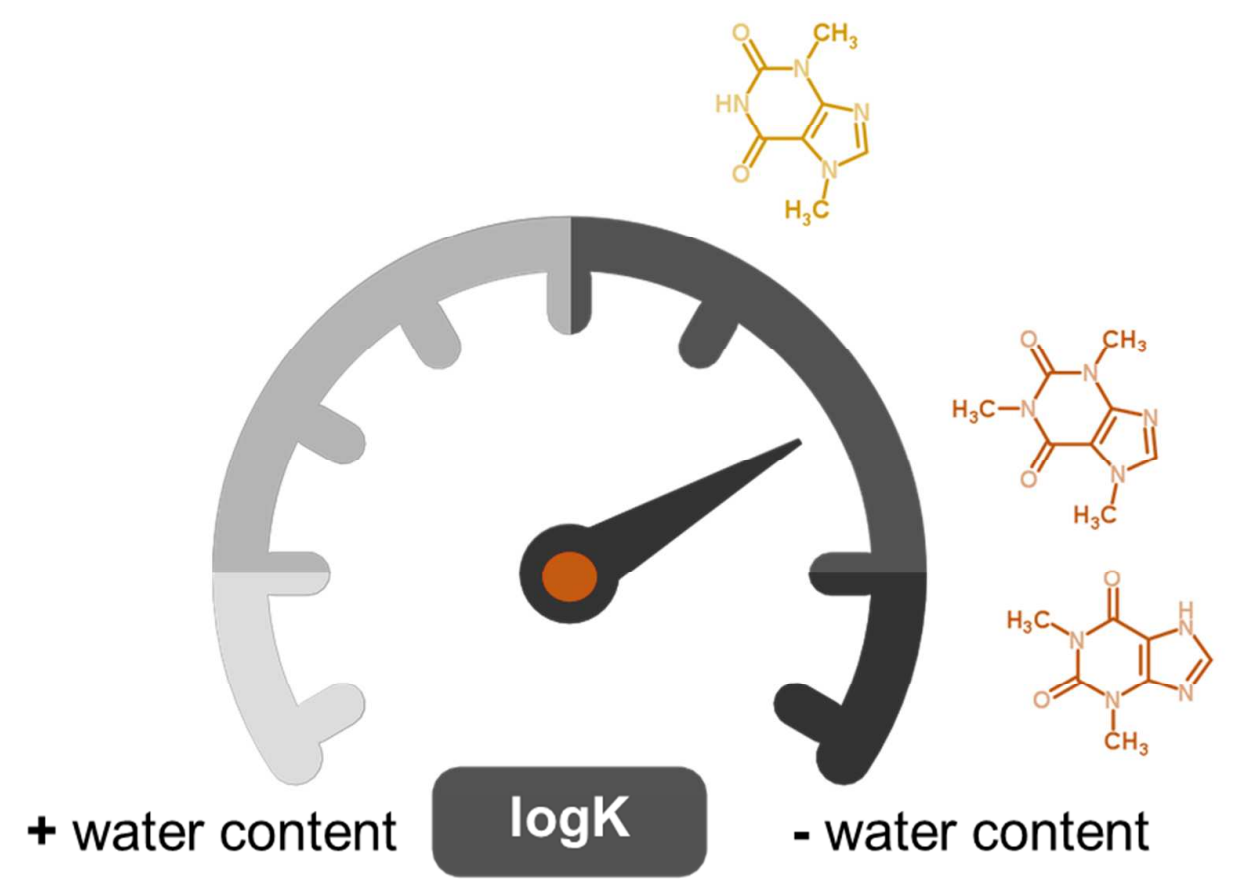

\section{Synopsis}

The use of alkaloids partitioning is used to evaluate the relative hydrophobicity of the phases in biocompatible cholinium-based Aqueous Biphasic Systems. 